\title{
Guest editorial to the special issue internal waves in the oceans and estuaries: modeling and observations
}

\author{
A. D. Rao $\cdot$ S. Hamdi
}

Received: 26 January 2011/Accepted: 26 January 2011/Published online: 15 February 2011

(C) Springer Science+Business Media B.V. 2011

Internal waves that owe their existence to density/gradients setup by temperature and/or salinity fields play an important role in ocean dynamics. Whereas the application of internal waves in Naval Warfare is well known, what is not well documented is their role in Natural Hazards. Internal waves can augment surface waves through coupling, and when such a coupling occurs to storm surges and tides, there could be an increasing risk of intensified coastal inundation due to tropical and extra-tropical cyclones and tsunamis. Internal waves, under the right conditions, lead to the so-called Dead Water phenomena and can seriously hamper navigation. Such incidents in the world's shipping lanes are well documented.

We thank Springer Publishers and the editorial staff of Natural Hazards for inviting us to guest edit this special issue, which contains a total of eight papers. The first paper by S. V. Babu and A. D. Rao deals with the dynamics of mixing due to internal waves in the north western part of the Bay of Bengal during winter period. The second paper by Sridevi et al. is about the impact of internal waves on the acoustic field. Paper three is authored by Rao et al. and explains how stratification affects thermal inversions. A more theoretical approach was taken in the fourth paper by Hamdi et al. and examines analytical solutions and conservation laws for long nonlinear internal waves.

The theoretical approach is continued in paper five by the same authors, where they employed method of lines simulations of the extended Korteweg-de Vries equation. Craig et al. deal with the coupling of internal waves and surface waves in paper six. Hareesh Kumar et al. describe in paper seven, how low-frequency internal waves influence the transmission loss variability. The final paper, written by Prasad and Rajasekhar,

\footnotetext{
A. D. Rao ( $($ )

Centre for Atmospheric Sciences, Indian Institute of Technology Delhi, Hauz Khas,

New Delhi 110 016, India

e-mail: adrao@cas.iitd.ernet.in

S. Hamdi

Solid Mechanics Laboratory (LMS), Ecole Polytechnique, 91128, Palaiseau Cedex, Paris, France

e-mail: samir.hamdi@utoronto.ca
} 
observation provides data on internal waves in the northern part of the Bay of Bengal using space borne SAR.

We hope that this collection of eight papers in this special issue will contribute to the ever growing knowledge of the role of internal waves in the oceanic processes. 